\title{
A comparison of forward and backward masking'
}

\author{
PETER H. SCHILLER AND MARILYN C. SMITH
}

MASSACHUSETTS INSTITUTE OF TECHNOLOGY

\begin{abstract}
Differences between forward and backward masking were studied by employing a disk, a ring and a pattern as masking stimuli and letters as test stimuli. The results show that interference is greater in forward than in backward masking. Interference was found to decrease monotonically with increasing interstimulus intervals (ISI) in all cases. Of the three masking stimuli, the ring produced the least interference and the pattern the most.
\end{abstract}

\section{Problem}

The recent series of experiments on visual masking (Eriksen \& Hoffman, 1963; Eriksen \& Lappin, 1964) has led to the suggestion that masking is due to luminance summation and contrast reduction. Proof for this view is seen in the finding that the masking effect is the same when the masking stimulus precedes the test stimulus as when it follows it (Eriksen \& Lappin, 1964). However, this symmetry between forward masking (FM) and backward masking (BM) may not be a general rule; the present study was therefore undertaken to extend the range of conditions under which forward and backward masking could be compared.

In the investigation of BM typically two sets of functions have been obtained. With overlapping test and masking stimuli a monotonically decreasing function has been reported, i. e., masking diminishes as the interstimulus interval (ISI) is increased (Kolers, 1962; Schiller, 1965). With stimuli having adjacent contours a U-shaped curve has frequently been obtained, i. e., masking "first grows" with increasing ISI, reaches a maximum at some intermediate ISI, and then declines again as ISI is further increased (Alpern, 1953; Kolers \& Rosner, 1960; Werner, 1935). However, with certain psychophysical procedures such a U-shaped function was not found, even though non-overlapping stimuli were used (Eriksen \& Collins, 1964; Fehrer \& Raab, 1962; Schiller \& Smith, 1965). A further aim of the present study was therefore to determine what type of masking function is obtained using two different psychophysical procedures.

\section{Method}

Two Ss with 20/20 vision were employed. All stimuli were presented in a three-field tachistoscope which was driven by a Sky Instrument Company programmer. The test stimuli were negative prints of No. 118 Futura Medium "Lettraset" capital letters, subtending a visual angle of $40^{\prime}$; they were illuminated from behind, so that Ss saw a light letter on a black background. The masking stimuli, shown in Fig. 1, consisted of (1) a ring (visual angle $\left.=65^{\prime}\right)$, (2) a disk (visual angle $=45^{\prime}$ ), and (3) a

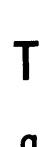

a
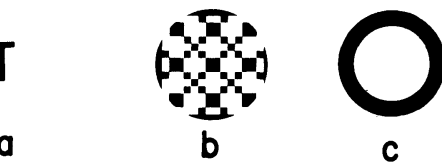

c

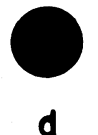

Fig. 1. (a) Sample of a test stimulus; (b) (c) (d) masking stimuli. circular pattern (visual angle $=65^{\prime}$ ). The masking stimuli, which were also negative prints, transmitted an equal amount of luminance flux; their luminance was $4.2 \mathrm{ft} \mathrm{L}$. The duration of the letters and masking stimuli was held constant at $2 \mathrm{msec}$. At each ISI employed $(5,30,60$, and $100 \mathrm{msec}$.) the intensity of the letter was increased in $0.1 \mathrm{log}$ unit steps by the use of Kodak neutral density filters until Ss correctly identified the letter. Stimuli were presented to the dark adapted eye.

Half of each session was devoted to FM, and the other half to BM, with the order reversed each day. Masking stimuli were randomized in blocks of 8 , ISIs in blocks of 4 , with thresholds for 2 letters taken at each ISI per session. Over a period of 10 sessions 20 thresholds were obtained for each of the 24 conditions.

\section{Results}

The results for two Ss, illustrated in Fig. 2, show the following; (1) The amount of interference is greater with FM than with BM. Analysis of variance showed this difference to be significant beyond the .01 level. The data indicate that the differences are slight at the short ISIs but increase with increasing ISI. The interaction between ISI and masking order was also significant for

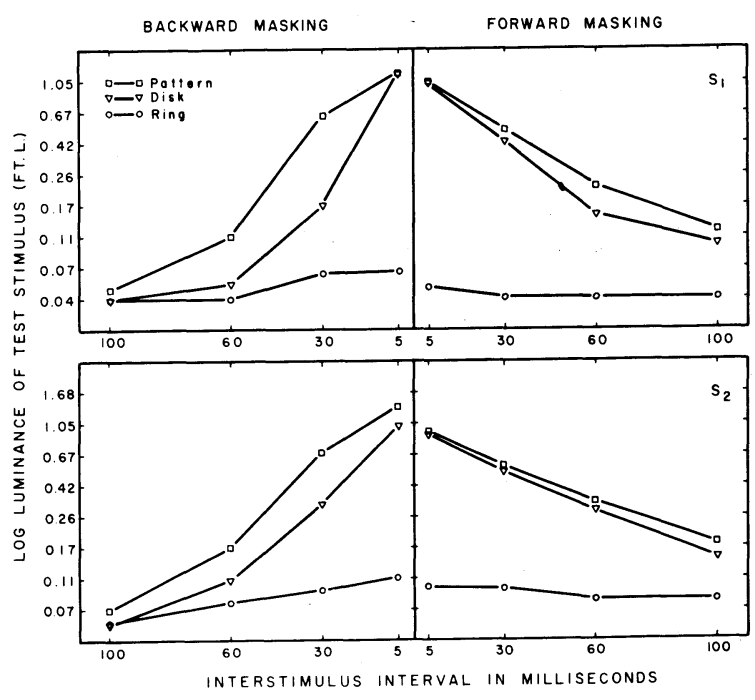

Fig. 2. Recognition thresholds for forward and backward masking as a function of masking stimuli and interstimulus intervals. 
TABLE I

PER CENT CORRECT DETECTION FOR LETTERS FOLLOWED BY A RING

RING LUMINANCE 42 FT L.

\begin{tabular}{lrrrrrrr} 
& \multicolumn{8}{c}{ ISI in msec. } \\
& & 3 & 20 & 40 & 60 & 100 & 140 \\
\cline { 2 - 8 } & 10 & 13 & 12 & 13 & 51 & 64 \\
$\begin{array}{l}\text { Intensity } \\
\text { of letter }\end{array}$ & .42 & 100 & 100 & 100 & 100 & 100 & 100
\end{tabular}

both Ss $(p=.01)$. (2) Patterns were found to mask to a greater extent than flashes; this difference was larger in BM than in FM. Such differences have been noted earlier (Schiller, 1965). The ring produced little masking relative to the pattern and flash. The differences between masking stimuli were significant for both Ss $(p=.01)$. (3) None of the masking stimuli yielded a Ushaped curve. A decreasing monotonic function with increasing ISI was obtained in all cases.

As it has been suggested that different psychophysical rocedures may yield different functions in BM when the test and masking stimuli do not overlap, in a second experiment we tried to determine whether, with a ring as a mask, a U-shaped curve would resultusing a method of constant stimuli. Two luminance levels were employed: 4.2 and $.42 \mathrm{ft} \mathrm{L}$. The luminance of the ring was constant at $42 \mathrm{ft} \mathrm{L}$. The duration of both the test letters employed and the ring was $5 \mathrm{msec}$.At each ISI condition $(3,20,40,60,100$ and $140 \mathrm{msec}),$.128 measures were taken. An example of the results for a typical $\mathrm{S}$ shown in Table 1, once again does not show a U-shaped function.

\section{Discussion}

The differences obtained between FM and BM suggest that the processes underlying them are not identical. It would seem that in FM there must at least be an added component involved, the exact nature of which remains to be determined. This component may be similar to a figural after-effect or a strong after-image brought about by the masking stimulus.

Similar differences in FM and BM have been reper $d$ by Sperling (1960). In other studies we have fou r lat when one employs the method of increasing ISI 11 the test stimulus is identified $s$ an ISI of almost tw e the duration is generally required for recognition in FM.
Our failure to obtain a U-shaped curve suggests, as do the findings of other studies (Fehrer \& Raab, 1962; Schiller \& Smith, 1965) that interference of this sort does not reflect a process involving "erasure" (Averbach \& Coriell, 1961). We have found that in some cases, with a method of constant stimuli, naive Ss show a Ushaped curve; this, however, disappears rapidly with practice.

Finally, results show that the extent of masking is dependent on the type of masking stimulus used. Stimuli which do not overlap with the test stimulus produce relatively little interference. The amount to which overlapping stimuli mask depends on their figural characteristics. In other studies it has been shown that flashes mask only monoptically, while patterns produce monoptic and dichoptic masking (Schiller, 1965; Sperling, 1963).

\section{References}

Alpern, M. Metacontrast. J. Opt.. Soc. Amer., 1953, 43, 648-657. Averbach, E., \& Coriell, A. S. Short-term memory in vision. Bell Sys. tech. J., 1961, 40, 309-328.

Eriksen, C. W., \& Collins, J. F. Backward masking in vision. Psychon. Sci., 1964, 1, 101-102.

Eriksen, C. W., \& Hoffman, M. Form recognition at brief durations as a function of adapting field and interval between stimulations. J. exp. Psychol., 1963, 66, 485-499.

Eriksen, C. W., \& Lappin, J. S. Luminance summation-contrast reduction as a basis for certain forward and backward masking effects. Psychon. Sci., 1964, 1, 313-314.

Fehrer, E., \& Raab, D. Reaction time to stimuli masked by metacontrast. J. exp. Psychol., 1962, 63, 143-147.

Kolers, P. A., \& Rosner, B. S. On visual masking (metacontrast) Res., 1962, ?, 777-294.

Kolers, P.. A., \& hosner, B. A. On visual masking (metacontrast): Dichoptic observation. Amer. J. Psychol., 1960, 73, 2-21.

Schiller, P. H. Monoptic and dichoptic visual masking by patterns and flashes. J. exp. Psychol., 1965, 69, 193-199.

Schiller, P. H., \& Smith, M. C. Detection in metacontrast. J. exp. Psychol., in press.

Sperling, G. Negative afterimage without prior positive image. Science, 1960, 131, 1613-1614.

Sperling, G. What visual masking can tell us about temporal factors in perception. Proc. XVII internat. Congress Psychol., 1963, 199-200.

Werner, H. Studies on contour: I. Qualitative analysis. Amer. J. Psychol., 1935, 47, 40-64.

Note

1. This research was supported by the National Institute of General Medical Sciences Grant T1 GM-1064. 